\title{
Caecal and sigmoid volvulus in the same patient
}

\author{
P. G. REASBECK* \\ M.R.C.P., F.R.C.S.
}

Royal Hampshire County Hospital, Winchester

\begin{abstract}
Summary
A patient is described who developed caecal volvulus several years after sigmoid colectomy for recurrent sigmoid volvulus. The pathophysiology of colonic volvulus is briefly reviewed, and it is suggested that an innate tendency to poor muscular tone in the colon may predispose to volvulus, while conversely excessive tone may lead to diverticular disease.

\section{Introduction}

In the United Kingdom volvulus of the sigmoid colon is an uncommon cause of large bowel obstruction; caecal volvulus occurs even more rarely. The development of both varieties in the same patient does not seem to have been reported previously.
\end{abstract}

\section{Case history}

The male patient (then aged 38 years) first presented in 1948 after an episode of lower abdominal pain and vomiting lasting several hours. Three similar attacks had occurred in the previous 14 years, 2 of which had required hospital admission. Physical examination was unremarkable but a barium enema showed a mobile caecum attached to a mesentery and a long redundant sigmoid loop, suggesting the possibility of recurrent volvulus. No treatment was given at that time.

In 1971 he was admitted as an emergency with acute large bowel obstruction. A diagnosis of sigmoid volvulus was made on the radiological appearances and the colon decompressed by sigmoidoscopy. Two months later he required further admission for another episode of sigmoid volvulus, again treated conservatively by sigmoidoscopy, and one week later an elective sigmoid colectomy was performed.

He made an uneventful recovery and was asymptomatic until September 1977 when he was admitted with acute large bowel obstruction of $24 \mathrm{hr}$ duration. Sigmoidoscopy showed no abnormality, apart from a rather distended rectum. Plain X-rays revealed a caecal volvulus and laparotomy was performed. At

\footnotetext{
*Present address: Dept of Surgery, University of Otago, P.O. Box 913, Dunedin, New Zealand.
}

operation the diagnosis of caecal volvulus was confirmed. Apart from considerable mobility of the caecum no underlying cause for the volvulus was apparent, although the whole large bowel was noted to be rather atonic and distended. The volvulus was reduced and a caecostomy performed.

Postoperatively he developed a clostridial septicaemia which responded to antibiotic treatment but otherwise recovery was uneventful. A plain X-ray taken 3 weeks postoperatively, just before his discharge, revealed persistent distension of the large bowel, although at this time he was well and asymptomatic. Six weeks postoperatively he was readmitted with a further episode of caecal volvulus which subsided spontaneously. Following this he has remained well.

\section{Discussion}

A number of local factors, particularly abnormal mesenteric attachment or abnormal mobility, are known to predispose towards caecal volvulus (Dowling and Gunning, 1969), but it has been pointed out (Anderson, Bergdale and van der Linde, 1975) that free mobility of the caecum is frequent in normal individuals (occurring in about $10 \%$ of post-mortem specimens) and therefore hypofixation alone does not suffice as an explanation for caecal volvulus.

Colonic distension, either associated with dietary factors, distal obstruction, or as a manifestation of one of the various forms of megacolon, is well known to predispose towards volvulus. Shepherd (1968) has suggested that a certain amount of torsion, unaccompanied by obstruction, may be a normal variant. He has pointed out that the presence or onset of distension in such a twisted segment could increase angulation at the end of the loop sufficiently to precipitate acute obstruction; in other words, it may be the distension rather than torsion per se which is the direct antecedent of acute obstruction. It seems reasonable therefore to suppose that the episodes of volvulus of 2 parts of the colon in the patient described here were associated with the persistent colonic distension demonstrated following 
his recovery. Shepherd (1969) noted a similar tendency for colonic distension to persist following resolution of sigmoid volvulus in Africans, and further observed that the degree of distension did not increase progressively with recurrent episodes of volvulus, suggesting again that this distension was primary and not secondary to the volvulus.

Thus, an unusual degree of colonic dilatation, not associated with any demonstrable pathology and therefore presumably representing one extreme of the normal range, may possibly predispose towards colonic volvulus. The author was unable to obtain any evidence of unusual dietary habits, undue constipation, purgative abuse or obstructive pathology in his patient, and must therefore invoke other factors to explain this distension. It may be that the degree of muscular tone in the colon is partly an innate (and therefore probably inherited) characteristic. Thus, those individuals with excessive tone or activity would be at risk of developing diverticular disease, while those with poor tone would tend to develop colonic distension, and a predisposition towards large bowel volvulus. This hypothesis would explain the observed inverse relationship between the geographical distribution of diverticular disease and colonic volvulus. It would also explain Shepherd's observation (1969) of a very marked tribal variation in the incidence of sigmoid volvulus in Central Africa. Support for the converse possibility of an innate abnormality in muscle tone underlying diverticular disease comes from a recent report (Rees and Griffin, 1977) of severe sigmoid diverticular disease in a 9-year-old child, in whom dietary factors presumably would not have had time to act.

\section{Acknowledgment}

I wish to thank Mr J. S. Mousley for permitting me to treat and report on a patient under his care, and for his advice in preparing this paper.

\section{References}

Anderson, A., Bergdale, L. \& VAN Der Linder, W. (1975) Volvulus of the caecum. Annals of Surgery, 181, 876.

Dowling, B.L. \& GunNing, A.J. (1969) Caecal volvulus. British Journal of Surgery, 56, 124.

ReES, B.T. \& GriffiN, P.J.A. (1977) Colonic diverticulosis in a child. British Medical Journal, 2, 1194.

ShePHERD, J.J. (1968) Treatment of volvulus of the sigmoid colon: a review of 425 cases. British Medical Journal, 1, 280.

ShEPHERD, J.J. (1969) The epidemiology and clinical presentation of sigmoid volvulus. British Journal of Surgery, 56, 353. 